\title{
EFFECT OF THE SIDE WALLS ON THE UNSTEADY FLOW IN A DUCT OF RECTANGULAR CROSS-SECTION
}

\author{
M. Emin Erdogan, C. Erdem Imrak \\ Faculty of Mechanical Engineering, Istanbul Technical University, 34439 \\ Gumussuyu, Istanbul, Turkey.imrak@itu.edu.tr
}

\begin{abstract}
In this paper, the effects of the side walls on the unsteady flow in a duct of rectangular cross-section are considered. In order to show the effects of the side walls on the flow, two illustrative examples are given. One of them is the unsteady flow due to an applied pressure gradient in a duct of rectangular cross-section. The other is the unsteady flow in a duct of rectangular cross-section moving parallel to its length. These effects depend on the values of the aspect ratio. The variations of the velocity and the volume flux with respect to the aspect ratio show that these are appreciable effects of the walls on the flow. It is found that the required time to reach the steady-state for the flow with side walls is shorter than that for the flow without side walls.
\end{abstract}

Keywords- Unsteady flow, duct of rectangular cross-section, effect of side walls, steady-state

\section{INTRODUCTION}

Effects of the side walls and edge effects occurring when the fluid flows in a finite domain have been investigated by many authors. The effect of the side walls on steady flow have been studied by Erdoğan $[1,2]$. The flows between disks of large but finite radii were examined by Brady and Durlofsky [3]. They showed that the effects of the end are not confined to a region near the end. The flow of an elastico-viscous liquid due to a rotating disk have been examined by Griffiths et al. [4] and they showed that edge effects can have quite a strong influence on the flow characteristics. Griffiths and Walters [5] noted that edge effects affect the whole of a flow field.

In this paper, effects of the side walls on the unsteady flow in a duct of uniform cross-section produced by an applied pressure gradient or by impulsive motion of a boundary are investigated. In order to show the effects of the side walls on the unsteady flow in a duct of uniform cross-section, two illustrative examples are given. One of them is the unsteady flow in a duct of rectangular cross-section moving parallel to its length and the other is the unsteady flow in a duct of rectangular crosssection due to an applied pressure gradient. The unsteady flow in a duct of rectangular cross-section have been investigated by many authors. Fan and Chao [6] have studied the flow in a duct of rectangular cross-section for various boundary and initial conditions. Drake [7] has investigated the flow in a duct of rectangular cross-section due to periodic pressure gradient. Erdoğan [8] has investigated the starting flow in a duct of rectangular cross-section due to an applied pressure gradient and has compared it with the starting flow between two parallel plates.

The fluid in a duct of rectangular cross-section where sides are at rest starts by sudden application of a constant pressure gradient. The solution of the governing equation for an incompressible viscous fluid may be obtained by a method which can be applied to all unsteady unidirectional flows $[9,10]$. The solution has two parts: the first part corresponds to the solution for the steady-state and the second shows the deviation 
from the steady-state. The solution has two non-dimensional parameters. One of them is the non-dimensional time $v t / b^{2}$ and the other is the aspect ratiob/a; where $v$ is the kinematic viscosity of the fluid, $t$ is time and, $2 a$ and $2 b$ are the length of the sides of cross-section of rectangular duct. The first term in the time-dependent part survives longest; therefore, the first term dominates the series. The expression is obtained for large times however, it can also be used for small values of the time, if a sufficient number of terms are considered [11]. For $v t / b^{2}=0.5$, the volume flux across a plane normal to the flow for a duct of square cross-section, $b / a=1$, is about 0.8394 and the volume flux for the flow between two parallel plates, $b / a=0$, is about 0.7180 .

In order to show the effect of the side walls on the unsteady flow the second example is the unsteady flow in a duct of rectangular cross-section moving parallel to its length. The results obtained are compared with those of the unsteady flow between two parallel plates suddenly set in motion with the same speed along the flow. For $v t / b^{2}=0.5$, the volume flux across a plane normal to the flow for a duct of square cross-section, namely $b / a=1$, is about 0.8086 and the volume flux for a duct in the absence of the side walls, namely $b / a=0$, is about 0.7639 . If the non-dimensional time is fixed, when the aspect ratio increases, the volume flux increases due to the effect of the side walls.

\section{UNSTEADY FLOW IN A DUCT OF RECTANGULAR CROSS-SECTION}

Consider the flow of an incompressible viscous fluid in a duct of rectangular cross-section whose sides are at $x= \pm a$ and $y= \pm b$ as shown in Figure 1 and it is assumed that $a \geq b$ throughout the paper. The governing equation is

$$
\frac{\partial w}{\partial t}=-\frac{1}{\rho} \frac{d p}{d z}+v\left(\frac{\partial^{2} w}{\partial x^{2}}+\frac{\partial^{2} w}{\partial y^{2}}\right),
$$

where $w(x, y, t)$ is velocity, $v$ is the kinematic viscosity of the fluid, $\rho$ is the density of the fluid, $d p / d z$ is an applied pressure gradient and $x, y, z$ are coordinates. The boundary and initial conditions are

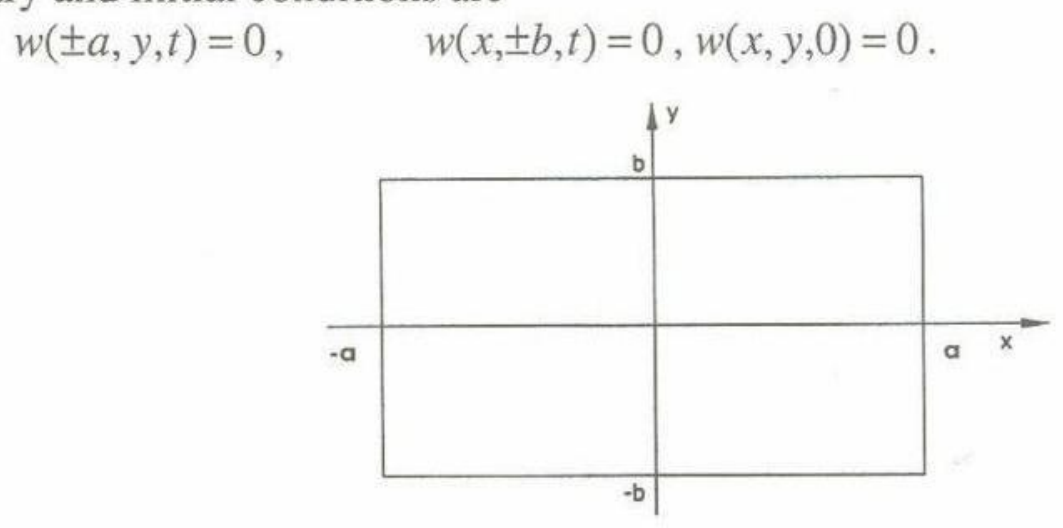

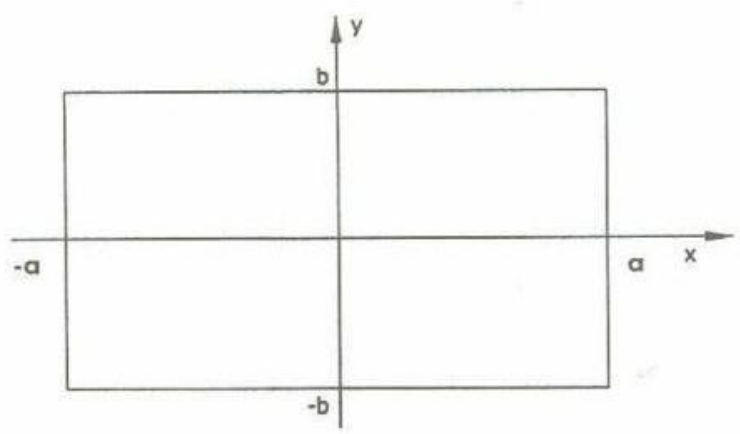

Figure 1. The coordinate systems for a duct of rectangular cross-section

The fluid starts with sudden application of a constant pressure gradient. Using the method given in $[9, .10]$, the solution subject to the conditions (2) can be found in the following form 


$$
\begin{aligned}
\frac{w(x, y, \tau)}{w(0,0, \infty)}= & {\left[1-\frac{32}{\pi^{3}} \sum_{n=0} \frac{(-1)^{n}}{(2 n+1)^{3}} \frac{1}{\cosh \lambda_{n} a}\right]^{-1}\left\{1-\frac{y^{2}}{b^{2}}-\frac{32}{\pi^{3}} \sum_{n=0} \frac{(-1)^{n}}{(2 n+1)^{3}} \frac{\cosh \lambda_{n} x}{\cosh \lambda_{n} a} \cos \lambda_{n} y\right.} \\
& \left.-\sum_{p=0} \sum_{q=0} A_{p q} e^{-b^{2}\left(\mu_{p}^{2}+\lambda_{q}^{2}\right) \tau} \cos \mu_{p} x \cos \lambda_{q} y\right\}
\end{aligned}
$$

where

$$
A_{p q}=\frac{128}{\pi^{4}} \frac{(-1)^{p+q}}{(2 p+1)(2 q+1)^{3}}\left[1-\frac{b^{2} / a^{2}}{b^{2} / a^{2}+(2 q+1)^{2} /(2 p+1)^{2}}\right],
$$

and $\mu_{p}=(2 p+1) \pi / 2 a, \lambda_{q}=(2 q+1) \pi / 2 b, \tau=v t / b^{2}$. When $\tau$ goes to infinity equation (3) reduces to

$$
\frac{w}{-\frac{1}{2 \mu} \frac{d p}{d z} b^{2}}=1-\frac{y^{2}}{b^{2}}-\frac{32}{\pi^{3}} \sum_{n=0} \frac{(-1)^{n}}{(2 n+1)^{3}} \frac{\cosh \lambda_{n} x}{\cosh \lambda_{n} a} \cos \lambda_{n} y
$$

which is the velocity for the unsteady-state in a duct of rectangular cross-section. The first two terms in equation (3) show the velocity in the case of the steady-state in a duct of rectangular cross-section. The term of double sum is the part of time-dependent of the velocity. The first term in the part of time-dependent dominates the series. When the aspect ratio goes to zero, equation (3) reduces to

$$
\frac{w}{-\frac{1}{2 \mu} \frac{d p}{d z} b^{2}}=1-\frac{y^{2}}{b^{2}}-\frac{32}{\pi^{3}} \sum_{n=0} \frac{(-1)^{n}}{(2 n+1)^{3}} e^{-(2 n+1)^{\frac{2^{2}}{4} \tau}} \cos (2 n+1) \frac{\pi}{2} \frac{y}{b}
$$

which is the velocity for unsteady flow between two parallel plates. In this case, there is no effect due to the side walls. The variation of the velocity at $x=0$, with respect to $y / b$ for various values of $b / a$ and $v t / b^{2}$ is illustrated in Figure 2 .

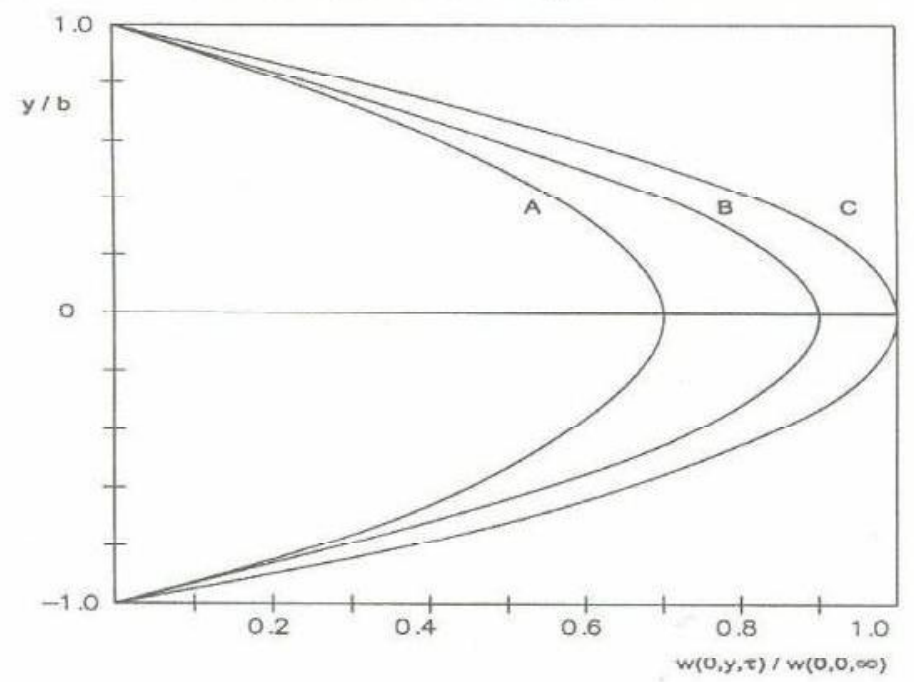

Figure 2. The variation of $w(0, y, \tau) / w(0,0, \infty)$, at $x=0$, with respect to $y / b$ for various values of $b / a$ and $v t / b^{2}$. A: $b / a=0, v t / b^{2}=0.5 ; \mathrm{B}: b / a=1, v t / b^{2}=0.5$; C: $b / a=0, v t / b^{2}=\infty$. 
The volume flux across a plane normal to the flow is

$$
\begin{aligned}
\frac{Q(\tau)}{Q(\infty)}= & -\frac{768}{\pi^{6}}\left[1-\frac{192}{\pi^{5}} \frac{b}{a} \sum_{n=0} \frac{\tanh \lambda_{n} a}{(2 n+1)^{5}}\right]^{-1} \sum_{p=0} \sum_{q=0} \frac{1}{(2 p+1)^{2}(2 q+1)^{4}}[1- \\
& \left.-\frac{b^{2} / a^{2}}{\frac{b^{2}}{a^{2}}+\frac{(2 q+1)^{2}}{(2 p+1)^{2}}}\right] e^{-b^{2}\left(\mu_{p}^{2}+\lambda_{q}^{2}\right) \tau},
\end{aligned}
$$

where

$$
\frac{Q(\infty)}{-4 a b^{3}(d p / d z) / 3 \mu}=1-\frac{192}{\pi^{5}} \frac{b}{a} \sum_{n=0} \frac{\tanh \lambda_{n} a}{(2 n+1)^{5}}
$$

which corresponds to the volume flux in the case of the steady-state. When $b / a$ goes to zero, equation (4) reduces to

$$
\frac{Q(\tau)}{Q(\infty)}=1-\frac{96}{\pi^{4}} \sum_{n=0} \frac{1}{(2 n+1)^{4}} e^{-(2 n+1)^{2} \frac{\pi^{2}}{4} \tau}
$$

which corresponds to the volume flux for the flow between two parallel plates. For $v t / b^{2}=0.5$, the volume flux for a duct of square cross-section, $b / a=1$, is about 0.8394 and the volume flux for the flow between two parallel plates, $b / a=0$, is about 0.7180 . If the non-dimensional time is fixed, when the aspect ratio increases the volume flux increases due to the effect of the side walls.

\section{UNSTEADY FLOW IN A DUCT OF RECTANGULAR CROSS-SECTION MOVING PARALLEL TO ITS LENGTH}

Consider the flow of an incompressible viscous fluid in a duct of rectangular cross-section where sides are at $x= \pm a$ and $y= \pm b$ as shown in Figure 1 and it is assumed that $a \geq b$ throughout the paper. The governing equation is

$$
\frac{\partial w}{\partial t}=v\left(\frac{\partial^{2} w}{\partial x^{2}}+\frac{\partial^{2} w}{\partial y^{2}}\right)
$$

where the symbols are defined in the previous section. There is no applied pressure gradient and the fluid starts suddenly due to the motion of the duct parallel to its length. The boundary and initial conditions are

$$
\begin{aligned}
& w( \pm a, y, t)=W \text { and } \quad w(x, \pm b, t)=W \text { for } \quad t>0, \\
& w(x, y, 0)=0 \text { for }-a<x<a \text { and }-b<y<b \text {. }
\end{aligned}
$$

Using the method given in $[9,10]$, the solution subject to conditions (6) can be found in the following form

$$
\frac{w}{W}=1-\frac{16}{\pi^{2}} \sum_{p=0} \sum_{q=0} \frac{(-1)^{p+q}}{(2 p+1)(2 q+1)} e^{-b^{2}\left(\mu_{p}^{2}+\lambda_{q}^{2}\right) \tau} \cos \mu_{p} x \cos \lambda_{q} y .
$$


When the aspect ratio $b / a$ is equal to zero, equation (7) reduces to

$$
\frac{w}{W}=1-\frac{4}{\pi} \sum_{n=0} \frac{(-1)^{n}}{2 n+1} e^{-(2 n+1)^{2} \frac{\pi^{2}}{4} \tau} \cos (2 n+1) \frac{\pi}{2} \frac{y}{b}
$$

which is the velocity for the flow between two parallel plates moving along the flow. In this case, there is no effect of the side walls on the flow. When the aspect ratio $b / a$ is equal to unity, equation (7) shows the velocity of the flow in a duct of square crosssection. The variation of $w / W$, at $x=0$, with respect to $y / b$, for various values of $b / a$ and $v t / b^{2}$ is illustrated in Figure 3.

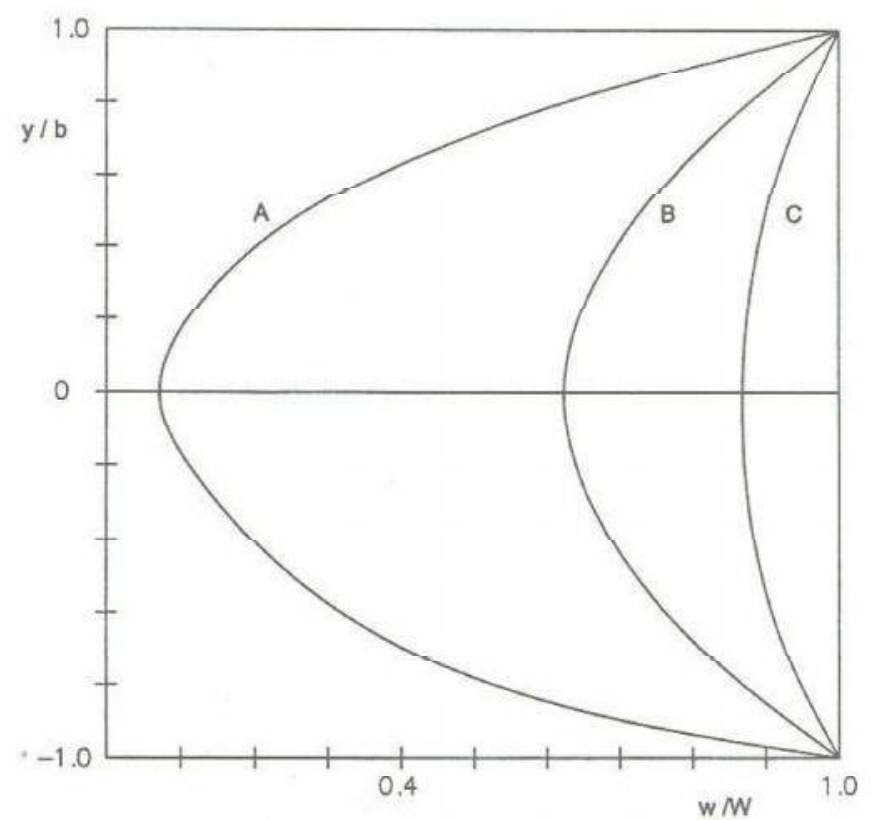

Figure 3. The variation of $w / W$, at $x=0$, with respect to $y / b$ for various values of $b / a$ and $v t / b^{2}$. A: $b / a=0, v t / b^{2}=0.1 ; \mathrm{B}: b / a=0, v t / b^{2}=0.5 ; \mathrm{C}: b / a=1$, $v t / b^{2}=0.5$.

The volume flux across a plane normal to the flow is

$$
\frac{Q}{4 a b W}=1-\frac{64}{\pi^{4}} \sum_{p=0} \sum_{q=0} \frac{1}{(2 p+1)^{2}(2 q+1)^{2}} e^{-\left[(2 p+1)^{2} \frac{b^{2}}{a^{2}}+(2 q+1)^{2}\right] \frac{\pi^{2}}{4} \tau} \text {. }
$$

If the aspect ratio $b / a$ goes to zero, equation (8) reduces to

$$
\frac{Q}{2 b W}=1-\frac{8}{\pi^{2}} \sum_{n=0} \frac{1}{(2 n+1)^{2}} e^{-(2 n+1)^{2} \frac{\pi^{2}}{4} \tau}
$$

which is the volume flux for the flow between two parallel plates moving along the flow. In this case, there is no effect due to the side walls. For $v t / b^{2}=0.5$, the volume flux for a duct of square cross-section, $b / a=1$, is about 0.8086 and the volume flux for the flow between two parallel plates, $b / a=0$, is about 0.7639 . If the non-dimensional time is fixed, when the aspect ratio increases the volume flux increases due to the effect of the side walls. 


\section{CONCLUSIONS}

Effects of the side walls on the unsteady flow in a duct of uniform cross-section produced by an applied pressure gradient or by impulsive motion of a boundary are investigated. In order to show the effects of the side walls on the unsteady flow in a duct of uniform cross-section two illustrative examples are given. One of them is the unsteady flow in a duct of rectangular cross-section moving parallel to its length and the other is the unsteady flow in a duct of rectangular cross-section due to an applied pressure gradient. It is found that the flow in a duct with side walls reaches steady-state earlier than the flow in a duct without side walls. This arises due to friction between the fluid and the wall.

\section{REFERENCES}

1. M.E. Erdoğan, Effects of the side walls on the flow over a moving plane wall, J. Appl. Mech. and Eng., 2, 415 - 432, 1997.

2. M. E. Erdoğan, Effects of the side walls in generalized Couette flow, J. Appl. Mech. and Eng. 3, 271 - 286, 1998.

3. J. F. Brady and L. Durlofsky, On rotating disk flow, J. Fluid Mech., 175, 363-394, 1987.

4. D. F. Griffiths, D. T. Jones and K. Walters, A flow reversal due to edge effects, J. Fluid Mech., 36, $161-175,1969$.

5. D. F. Griffiths and K. Walters, On edge effects on rheometry, J. Fluid Mech., 42, 379 $399,1970$.

6. C. Fan and B.-T. Chao, Unsteady, laminar, incompressible flow through rectangular ducts, ZAMP, 16, 351-360, 1965.

7. D. C. Drake, On the flow in a channel due to periodic pressure gradient, Quart. J. Mech. And Appl. Math., 18, 1 - 10, 1965.

8. M. E. Erdoğan, Starting flow in a channel of rectangular cross-section, Bull. Tech. Univ. Ist., 46 (1), 309-339, 1993.

9. M. E. Erdoğan, On the unsteady unidirectional flows generated by impulsive motion of a boundary or sudden application of a pressure gradient, Int. J. Non-Linear. Mech., 37, 1091 1106, 2002.

10. M. E. Erdoğan, On the flows produced sudden application of a constant pressure gradient or by impulsive motion of a boundary, Int. J. Non-Linear Mech., 38, 781 - 797, 2003.

11. M. E. Erdoğan and C. E. Imrak, On the unsteady flows generated by impulsive motion of a boundary, Math. \& Comput. Appl., 9 (2), 265 - 274, 2004. 